\title{
Risk of Infection after Deep Brain Stimulation Surgery with Externalization and Local-Field Potential Recordings: Twelve-Year Experience from a Single Institution
}

\author{
Lucia K. Feldmann $^{\mathrm{a}}$ Wolf-Julian Neumann ${ }^{\mathrm{a}}$ Katharina Faust ${ }^{\mathrm{b}}$ \\ Gerd-Helge Schneider ${ }^{b}$ Andrea A. Kühn ${ }^{a, c, d, e}$ \\ aDepartment of Neurology, Charité - Universitätsmedizin Berlin, corporate member of Freie Universität Berlin and \\ Humboldt-Universität zu Berlin, Berlin, Germany; ${ }^{\text {b} D e p a r t m e n t ~ o f ~ N e u r o s u r g e r y, ~ C h a r i t e ́ ~-~ U n i v e r s i t a ̈ t s m e d i z i n ~}$ \\ Berlin, corporate member of Freie Universität Berlin and Humboldt-Universität zu Berlin, Berlin, Germany; ${ }^{\circ}$ Berlin \\ School of Mind and Brain, Charité University Medicine, Berlin, Germany; dNeuroCure Clinical Research Centre, \\ Charité University Medicine, Berlin, Germany; ${ }^{e}$ DZNE, German Center for Degenerative Diseases, Berlin, Germany
}

\author{
Keywords \\ Deep brain stimulation · Infection · Externalization · Local \\ field potential
}

\begin{abstract}
Introduction: Deep brain stimulation (DBS) has been an established surgical procedure in the field of functional neurosurgery for many years. The experimental electrophysiological method of local field potential (LFP) recordings in postsurgically externalized patients has made substantial contributions to the better understanding of pathophysiologies underlying movement disorders. As interest in LFP recordings for the development of improved stimulation strategies increases, this study's aim was to provide evidence concerning safety of this research method, in a major DBS center. Methods: We retrospectively analyzed incidence and infection characteristics in adult patients who underwent two-staged DBS surgery with temporary externalization of leads in our center between January 2008 and November 2019. We focused on whether patients had participated in LFP recordings, and evaluated incidence of infections at 3 months and 1 year after the surgery based on medical re-
\end{abstract}

karger@karger.com www.karger.com/sfn

Karger $\frac{1}{\%}$
(C) 2021 The Author(s)

Published by S. Karger AG, Basel

This is an Open Access article licensed under the Creative Commons Attribution-NonCommercial-4.0 International License (CC BY-NC) (http://www.karger.com/Services/OpenAccessLicense), applicable to the online version of the article only. Usage and distribution for commercial purposes requires written permission. cords. Infection rates were compared to major DBS studies and reports focusing on the risk of infection due to externalization of DBS leads. Results were visualized using descriptive statistics. Results: Between January 2008 and November 2019, DBS surgery was performed in 528 patients (389/139 patients in the LFP/non-LFP group), mainly for movement disorders such as Parkinson's disease (308), dystonia (93), and essential tremor (86). Of the patients, $72.9 \%$ participated in LFP recordings. The incidence of infections in the acute postsurgical phase ( 3 months) was $2.46 \%$ and did not differ significantly between the LFP group (1.8\%) and the non-LFP group (4.32\%). The overall incidence after 1 year amounted to $3.6 \%$ (19 patients) with no difference between LFP/nonLFP groups. Incidence rates reported in the literature show a large variety $(2.6-10 \%)$, and the incidence reported here is within the lower range of reported incidences. Discussion/ Conclusion: This study demonstrates that DBS is a surgical procedure with a low risk of infection in a large patient cohort. Importantly, it shows that LFP recordings do not have

Andrea A. Kühn and Gerd-Helge Schneider contributed equally to the manuscript. 
a significant effect on the incidence of infections in patients with externalization. With a representative cohort of more than 380 patients participating in LFP-recordings, this underlines LFP as a safe method in research and supports further use of this method, for example, for the development of adaptive stimulation protocols. 02021 The Author(s).

Published by S. Karger AG, Basel

\section{Introduction}

Deep brain stimulation (DBS) is a well-established method improving motor symptoms and overall quality of life of patients suffering from movement disorders like Parkinson's disease (PD), dystonia, and essential tremor [1-5], but also in other indications such as psychiatric diseases [6]. Postoperative externalization of the DBS leads has been traditionally conducted as a method of quality control using external test stimulation and CT/ MRI imaging before the pulse generator is implanted. While many centers have extensive experience with externalization of DBS leads, neurosurgeons might be worried about a potentially increased risk of infection. Beside the benefits of test stimulation and imaging, externalization is of crucial importance for research as recordings using externalized leads provide the unique opportunity to record local field potentials (LFPs) directly from the basal ganglia in awake humans. This research has significantly contributed to the new concept of understanding movement disorders as network disorders [7-10]. It is of importance for further technical development of stimulation paradigms, for example, adaptive DBS $[11,12]$, requiring long recording sessions with postoperatively externalized patients. In research with postoperative patients, the patient safety must be of primary concern. In theory, manipulation of the sterile wound dressing in freshly operated patients and connection of the externalized DBS leads to a recording system for LFP-research could result in increased risk of infections. Infections have been regularly reported in major DBS studies (see Table 3) $[1,3-5,13-19]$ and incidence varies between 2.6 and $10 \%$. While there are a few studies investigating the risk of externalization of DBS leads [20-25], only 1 group has explored the incidence of infections in patients with perioperative LFP recordings showing that it was not increased [23].

Our center has been intensively conducting DBS research with more than 350 recordings in the last 10 years alone. There has been research for various indications, and using different DBS lead systems aiming to under- stand network disorders and identify biomarkers for future improved stimulation approaches such as closed loop DBS. To this end, LFP recordings get increasingly relevant for the development of these more sophisticated therapeutic approaches. Hence, it is of high importance to further investigate the impact of LFP recordings on infection rates in patients receiving two-staged DBS surgery with externalizations, and to evaluate the experience of a major DBS center.

\section{Materials and Methods}

\section{Data Acquisition}

In this study, we retrospectively included all adult patients who underwent DBS surgery with externalization in our center between January 2008 and November 2019 according to our database, accessed in November 2020. Medical records were evaluated for early surgery related infections at 3 months postimplantation and longer term infections at 12 months postimplantation and characterized according to the definition of the surgical site infection (SSI) [26]. Patients were grouped to whether they had received LFP recordings or not (the LFP group, the non-LFP group). If there had been an infection, we sought to illustrate the infection regarding the time course and treatment of the infection. The incidence of infections was calculated for the first 3 months postsurgery and the total amount of infections in the first postoperative year was assessed. We statistically compared the incidence rates between the LFP-recording and no LFP-recording group using the $\chi^{2}$ test for direct comparison, we further evaluated the risk difference (RD) and the evolution of infection risk over time between the groups with the log-rank test (MATLAB [Natick, MA, USA]/IBM SPSS [Armonk, NY, USA]).

\section{Surgical Procedure and Antibiotic Prophylaxis}

Depending on the indication for DBS surgery and the physical and psychological fitness of the patient, surgery was intended to be performed mainly under local anesthesia. Throughout the 12-year time span, the antibiotic prophylaxis regime changed. While initially ampicillin/sulbactam was applied intraoperatively, and then for 4 days following the procedure until 2010, this was changed toward peri-surgical single-shot antibiotic prophylaxis with cefuroxime since then. After premedication, the patient's hair was shaved and the scalp disinfected. Then, using regional anesthesia, the patient's head was placed in a stereotactic frame (RM-frame, inomed, Emmendingen, Germany/Leksell Frame, Elekta, Stockholm, Sweden). A CT-scan was performed for fusion of the preoperative MRI based stereotactic plan with the stereotactic CT. DBS electrode implantation followed stereotactic trajectory planning using a preoperative MRI in a commercially available planning software (STP4, Leibinger, Freiburg, Germany until 2016/Brain Lab, Munich, Germany). The surgery started in the hemisphere contralateral to the clinically more affected side; the patient was placed and draped in a way that face and eyes were situated on the non-sterile side of the drape and accessible for clinical investigation and anesthesiological procedures. After a frontoparietal curved incision, a burr hole of $14 \mathrm{~mm}$ diameter was prepared, the respective burr hole cover was inserted and fixed, dural cannulas 
for introduction were inserted. The microelectrode drive with up to 5 trajectories was positioned and used for exact controlled advance of the temporary test electrodes (Alpha Omega, Nazareth, Israel). Microelectrode recordings of multiunit activity in the last $10 \mathrm{~mm}$ above the target point were performed to assess firing patterns for electrophysiological determination of correct electrode position. In awake patients, symptoms as well as side effects were evaluated in test stimulation sessions for identification of the trajectory with best clinical effect/least side effect, using the Neuro Omega System (Alpha Omega, Nazareth, Israel). The trajectory was chosen according to the result of testing and the final DBS lead was inserted using fluoroscopic control of insertion depth. Between 2008 and 2019, DBS electrodes from different companies were used (Medtronic, Minneapolis, MN, USA; Boston Scientific, Marlborough, MA, USA; Saint Jude Medical and Abbott, Plano, TX, USA), depending on the patient preference, implantation time point, surgical target, indication, and availability of rechargeable or primary cell devices. The electrode was fixed in the manufacturers' burr hole cover, and extensions (Medtronic extension kit 3550-05, Boston Scientific 55 cm/8 Contact Extension Kit, St. Jude Medical Infinity ${ }^{\mathrm{TM}} \mathrm{DBS}$ system extension) for temporary externalization were connected. It was always taken care that externalization was performed at least at a distance of 6-7 cm from the original incision for the burr hole. Externalization was always done on the same side as the connection for the stimulator, which was performed in the second surgery. The procedure was repeated for the second hemisphere. On the day following surgery, a CT scan was performed to confirm correct electrode position and exclude postoperative hemorrhage concluding the first surgical stage. All patients were externalized with the intent to test the effect of stimulation and side effect thresholds without the restrictions of the operative setting.

The second stage of the DBS surgery was usually performed 2-6 days after the first procedure. Here, the externalized connections were removed and sterile lead extensions were tunneled subcutaneously. The neurostimulator was inserted in a subcutaneous pouch either in the abdominal region or infraclavicular and connected to the extensions. Before closure of skin, wounds were rinsed with antiseptic solution.

\section{LFP Recordings}

During the last 12 years, LFP recordings have been performed in our center for various experimental paradigms using stimulation, different motor, and cognitive tasks (e.g., [9, 27-38]); the technical setup has been constantly updated and improved. However, the general experimental procedure remained the same: during the externalization period, patients participated in LFP recordings in a dedicated electrophysiology laboratory. The experimental procedures were always conducted by a trained medical doctor, according to strict aseptic guidelines. Great care was taken in the handling of the sterile, postoperative wounds; wound dressings were removed, and wounds were cleaned with the antiseptic solution, then we placed a sterile preliminary dressing, including the DBS leads connected with the recording extension for the course of the recording session. Additional sensors for recording of electroencephalography, electromyography, or motor performance (e.g., accelerometer), as well as the ground electrode were attached; if necessary, for the experiment. Overall, LFP recordings are a complex procedure, which is more time-consuming than routine clinical test stimulation in externalized patients. After the record- ing, the wound was freshly dressed following renewed disinfection of the wounds applied.

\section{Literature Review}

We conducted a systematic literature search in PubMed regarding infections in DBS and movement disorders using the key terms (["parkinson's disease" OR “dystonia” OR "essential tremor"] AND "infection" AND ["deep brain stimulation” OR "deep brain" OR "DBS”] AND “externalization” OR "externalized” OR "externalizing") with 5 results in February 2021, and ("infection" AND ["deep brain stimulation" OR "deep brain" OR “DBS”] AND ["externalization" OR "externalized” OR “externalizing”]) yielding additional 2 references [20-25, 39], of which one was a review [25] and one was a case report [39]. Here, we additionally present our results in relation to the major controlled clinical DBS studies $[1,3-5,14,16-19,40-42]$ (Table 3 ). While a direct statistical analysis of literature proved difficult due to the variety of confounders, we conducted a descriptive confidence interval-based comparison of the studies.

\section{Results}

\section{Demographics}

Between January 2008 and November 2019, a total number of 528 adult patients received DBS surgery in our center, mostly for movement disorders such as PD (308), isolated and complex dystonia (93), essential tremor (86), as well as for other indications such as depression or symptomatic tremor. The main DBS targets were the subthalamic nuclei, internal pallidal globe, and ventral intermediate nucleus, for more characteristics see Table 1 . DBS leads manufactured by different companies were used in this study (Medtronic: 355 patients, Boston Scientific 147 patients, St. Jude Medical/Abbott: 26 patients).

\section{Incidence and Characteristics of Infections}

Within the 1-year follow-up period, infections occurred in $3.6 \%$ of cases (19/528), of which 13 manifested during the first 3 months (2.46\%). Eighteen out of 19 qualified as deep tissue incisional SSI. SSI provides a definition for postoperative infections including criteria such as incisional depth, time, symptoms, and infection characteristics; superficial SSI occur during the first 30 days involving only the skin and subcutaneous tissues, deep SSI occurs within 1 year for implants and includes deeper tissue layers [26]. For more details on the characteristics and timelines of the individual infections, please see online suppl. Table 1 (for all online suppl. material, see www.karger.com/doi/10.1159/000516150). Most infections were caused by bacteria classically resident on the skin such as Staphylococcus aureus (40.9\%), Staphylococcus epidermidis (13.6\%), Staphylococcus lugdunensis 
Table 1. Demographic overview

\begin{tabular}{lrrlll}
\hline & $N$ & $\begin{array}{l}\text { Mean age } \\
\text { (range), years }\end{array}$ & $\begin{array}{l}\text { Gender } \\
\text { distribution } \\
(\mathrm{f} / \mathrm{m})\end{array}$ & $\begin{array}{l}\text { Mean disease } \\
\text { duration (range) } \\
\text { years }\end{array}$ & $\begin{array}{l}\text { Mean period of } \\
\text { externalization, } \\
\text { days }\end{array}$ \\
\hline PD & 308 & $61.5(26-78)$ & $98 / 210$ & $11(1-45)$ & 6 \\
Dystonia & 93 & $51.3(18-79)$ & $54 / 39$ & $15(1-70)$ & 6 \\
ET & 86 & $68(25-83)$ & $42 / 44$ & $23(1-73)$ & 6 \\
Others & 41 & $50(20-75)$ & $18 / 23$ & $14(1-60)$ & 7 \\
STN & 288 & $60.9(20-78)$ & $90 / 198$ & $11(2-45)$ & 6 \\
GPi & 108 & $51(18-80)$ & $61 / 47$ & $14(1-70)$ & 6 \\
VIM & 109 & $66.3(24-83)$ & $51 / 58$ & $21(1-73)$ & 6 \\
Others & 11 & $56.3(24-76)$ & $5 / 6$ & $13(5-30)$ & 7 \\
2 sites & 12 & $56(39-75)$ & $5 / 7$ & $18(3-60)$ & 6 \\
\hline Total & 528 & $59.9(18-83)$ & $212 / 316$ & 14.2 & 6 \\
\hline
\end{tabular}

PD, Parkinson's disease; ET, essential tremor; STN, subthalamic nucleus; GPi, internal pallidal globe; VIM, intermediate ventral nucleus; $\mathrm{f} / \mathrm{m}$, female/male.

Table 2. Demographics LFP versus no LFP

\begin{tabular}{lcc}
\hline & $\begin{array}{l}\text { No LFP } \\
\text { recording }\end{array}$ & $\begin{array}{l}\text { LFP } \\
\text { recording }\end{array}$ \\
\hline Mean age (std), years & $63 \pm 12.9$ & $59 \pm 12$ \\
$\quad$ Range & $18-83$ & $20-80$ \\
Mean disease duration (std), years & $16.2 \pm 14$ & $13.38 \pm 10.5$ \\
Gender (f/m) & $63 / 76$ & $149 / 240$ \\
Mean externalization (std), days & $5.8 \pm 2.3$ & $6 \pm 1.6$ \\
\hline
\end{tabular}

LFP, local field potential recordings; std, standard deviation.

(9.1\%), Staphylococcus capitis (4.5\%), or the respiratory tract such as Streptococcus salivarius (4.5\%) and Moraxella olensis (4.5\%), as well as 1 case of Proteus mirabilis infection. In 3 cases, the relation of the SSI to a detectable infectious agent in microbiological examination was not successful. In 18/19 cases, either the neurostimulator (8) or DBS leads (3) or both (7) needed to be explanted. Calculated individualized antibiotic therapy was successful in all patients lasting between 1 week (superficial incisional infection) and 16 weeks (the mean duration of antibiotic therapy 6.4 weeks). Of the 18 patients, 11 received a reimplantation (DBS leads and neurostimulator: 4; neurostimulator: 4; DBS leads: 3) between 2 and 15 months later, without further complications. The remaining patients are planned to receive reimplantation, decided against reimplantation, or new contraindications had evolved in the meantime (e.g., percutaneous coro-

Incidence of Infections in DBS with Externalizations nary intervention with dual anticoagulant therapy) or were lost to follow-up. We also checked whether the change of the antibiotic regime (intraoperative application of Unacid until 2010 ( 2 infections/130 patients) versus peri-surgical treatment with cefuroxime from 2010 (17/398) had an impact on the infection rates; however, there was no significant difference in the $\chi^{2}$ test, $p=0.15$. Further, the infection rate was not related to the IPG implantation site (abdominal: 11/328, 3.4\%, infraclavicular $8 / 191,4.2 \%$, remaining patients lost to follow-up/information not retrievable; the $\chi^{2}$ test $p=0.6$ ).

\section{Infections and LFP Recordings}

Of all patients, 73.67\% (389/528) participated in LFP recordings, while $26.33 \%$ of the patients did not participate in postoperative recordings during the externalization period. There was no difference regarding demographic characteristics, as depicted in Table 2.

Importantly, there was no higher incidence of infections in the LFP group than in the non-LFP group (see Fig. 1). Most SSIs occurred during the first 90 days postsurgery. Statistical testing for the incidence of infections at 3 months after the surgery did not reveal significant differences between the patient groups (the LFP group: $1.87 \%$ (7/389) versus the non-LFP group $4.32 \%$ (6/139), the $\chi^{2}$-test: $p=0.1002$ ) (see Fig. 1, left). At 12 months postsurgery, there was also no significant difference in the groups (the LFP group 3.08\% (12/389) versus the nonLFP group $5.04 \%(7 / 139)$, the $\chi^{2}$-test: $\left.p=0.289\right)$. We also assessed the $\mathrm{RD}$, which is a comparative measure for risks 


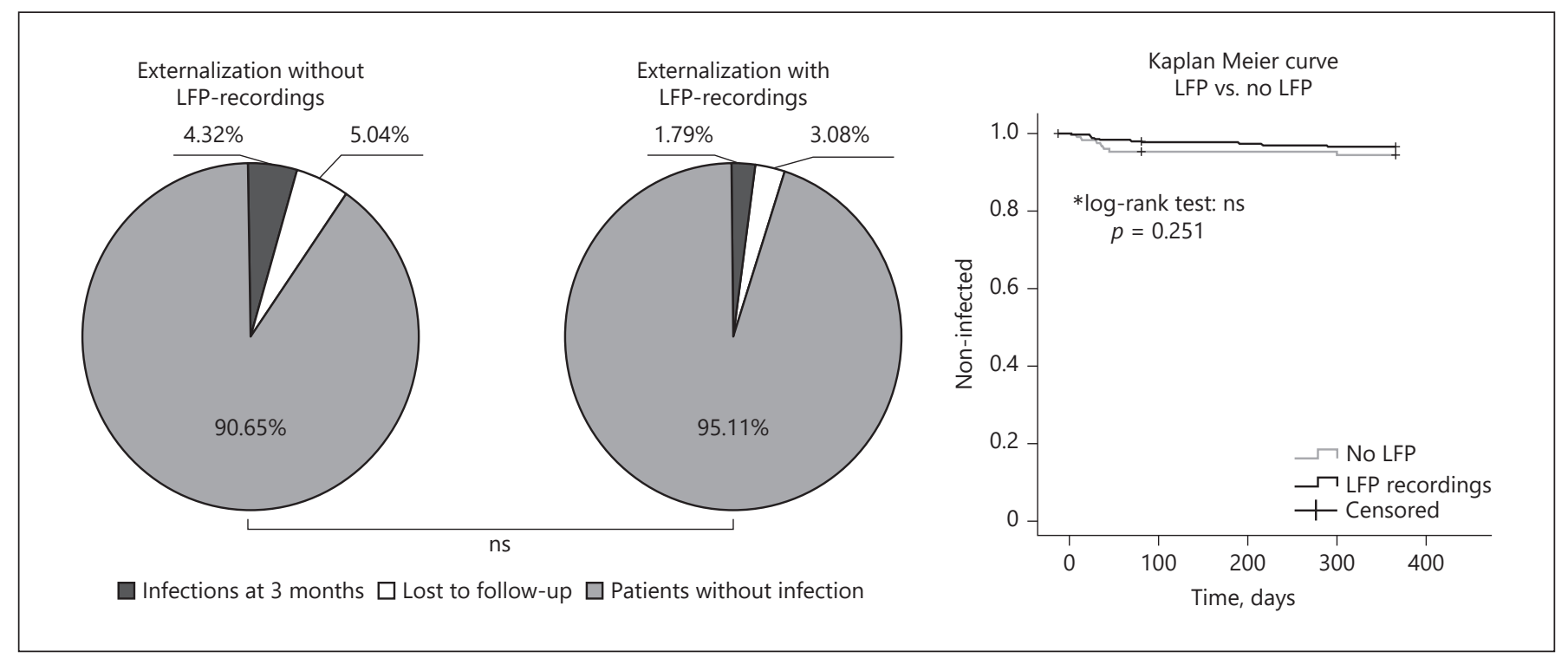

Fig. 1. Incidence of infections 3 months after surgery. Pie charts representing the incidence of infections in patients not participating in LFP-recordings (left pie chart) versus participation in LFP recordings (right pie chart) (left). Infections are represented in black, no reported infections in lighter gray. A minor proportion of patients were lost to follow-up (white). Very low incidences are reported for both patient groups, and there was no significant difference (the $\chi^{2}$ test $p=>0.05$ ) between the groups. Further statistical evaluation confirmed that there was no significant difference regarding infection onset over time (right) as depicted in the Kaplan-Meier Curve with a nonsignificant log-rank test $(p=>0.05)$ between the 2 groups. LFP, local field potential. (e.g., incidences) in different exposure groups, and is computed by subtraction of the incidence in the unexposed group from the incidence in the exposed group $(\mathrm{RD}=\mathrm{IE}-\mathrm{IU})$. Positive $\mathrm{RDs}$ indicate an increased risk, negative $\mathrm{RDs}$ indicate a decreased risk through exposure, the values range between 0 and \pm 1 [43]. Values close to zero indicate that the RD between the groups is low here, $\mathrm{RD}$ at 3 months $(-0.0237)$ and 12 months $(-0.018)$ was very low, implying no significant effect of LFP recordings on the risk of infection. There was also no significant difference in the risk of infection during the course of the follow-up time as depicted in the KaplanMeier Curve (Fig. 1, right) and objectified in a nonsignificant log-rank test statistical evaluation $(p=0.251)$.

\section{Literature Review}

The incidence of infections as a major adverse event is commonly reported in all prominent DBS studies. Our literature search in PubMed with key terms "infection," "deep brain stimulation," and "externalization" revealed 7 original articles. There was a grand variation regarding patient numbers, inclusion criteria of patients, and follow-up periods. Hence, to ensure comparison with a controlled, comparable study design, we here, additionally present our data in relation to the incidences for infections reported as serious adverse events and adverse events as events, or, where provided, per patient, in the major controlled clinical trials investigating DBS for each movement disorder individually (see Table 3 ) $[1,3-5,14-$ $19,40-42]$. The reported incidences ranged from $2.6 \%$ to up to $10 \%$. This study was in the lower range of the reported incidences. Due to a lack of uniform information on the surgical technique, externalization and conducted recordings or test-stimulations during the externalization period, time-point of the incidence analysis, and variable information on investigated indications we decided to refrain from statistical comparison but present the literature review descriptively for all studies with follow-up during the first 24 months post-surgery in Figure 2 , left. There was no significant trend for infections over time (Spearman correlation: $\rho=0.2348, p=0.176$ ). We further illustrated this study's position within the so far reported incidences, including the $95 \%$ confidence intervals in Figure 2, right, in a forest plot sorted by number of patients included in the study. This depicts the range of reported incidences and the strength of the findings depending on the reported case numbers showing that our results are representing a very large patient cohort at 


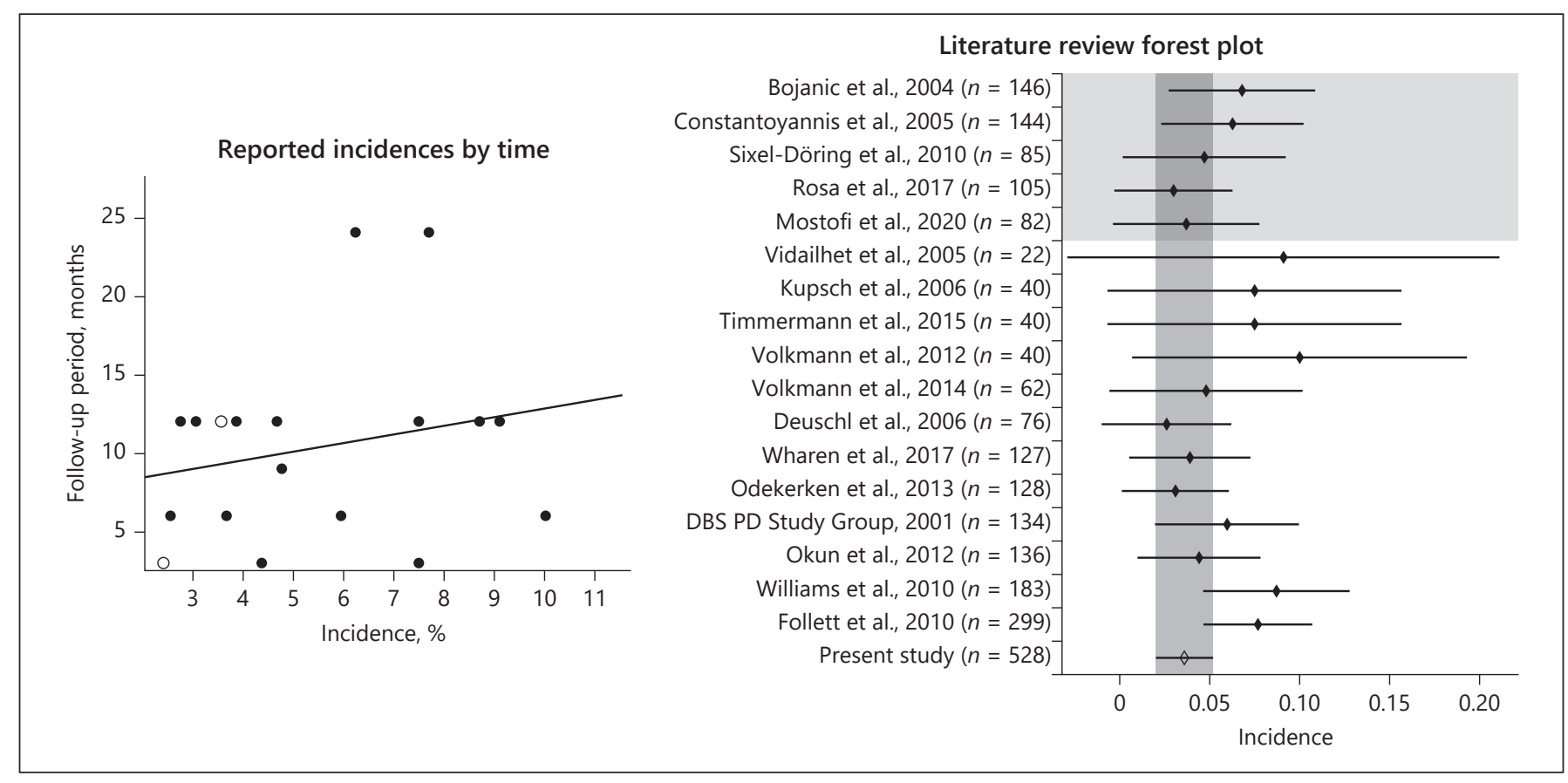

Fig. 2. The literature review for infection incidences reported in the literature review and in controlled clinical DBS trials during the first 24 months after surgery. Scatter plot representing all reported incidences (black scatter dots) by follow-up time (left), there was no significant trend $(\rho=0.2348, p=0.171)$. White scatter dots represent this study's incidences at 3 and 12 months postsurgery. Right: representation of the assessed studies in a forest plot (modified from a figure created using code available through MATLAB Central File Exchange [44]). Error bars represent the

a very robust low incidence rate in comparison with the previously published major controlled clinical DBS trials.

\section{Discussion/Conclusion}

Here, we retrospectively assess the potential association of increased risk of postsurgical infections with LFP recordings during temporal externalization of DBS electrodes in a large cohort of patients receiving DBS surgery in a single center. In a representative and large cohort of more than 500 patients with postoperative externalization of the DBS leads, there was no difference in the infection rate between patients with or without LFP recordings, neither at 3 months follow-up period nor at 12 months follow-up period. Moreover, the infection rate of $3.6 \%$ documented after 1 year was within the lower range of incidences reported in the literature so far. While most infections qualified as deep incisional SSIs and the DBSsystem had to be removed in most cases, the infections resolved without further complications under antibiotic therapy in all cases.

Incidence of Infections in DBS with Externalizations
95\% confidence intervals, the white incidence diamond represents the present study, the gray transparent bar aides the visualization of the present study's confidence intervals in relation to the other studies, the results of the literature review are presented with a gray background, and the results of the controlled clinical DBS trials on white background (DBS PD SG = deep brain stimulation for Parkinson's disease study group). DBS, deep brain stimulation; PD, Parkinson's disease; SG, study group.

\section{Reported Infection Rates in the Light of Incidences Reported in the Literature}

As can be seen in Figure 2, the incidences reported here were low in comparison with the major controlled clinical trials in DBS, with a range of incidences between 2.6 and $10 \%$. The high number of patients and this center's experience over a time span of $>10$ years, the use of DBS systems by different manufacturers and presentation of different indications for DBS surgery, make this a very robust finding. Here, opposed to most other studies, we exclusively investigated infection incidences in externalized patients. There has been an ongoing discussion in the field whether externalizations promote infections. In a recent systematic review, a meta-analysis revealed no difference in the infection rate between externalized (5.2\%) and non-externalized patients (6.0\%), but the authors were hesitant to draw conclusions due to the high heterogeneity between studies [25]. For instance, Constantoyannis et al. [21] report an increased infection risk with externalized leads, while 2 other studies reported no 
Table 3. Overview literature search (shaded gray)/major DBS studies (white) and reported incidences of infections

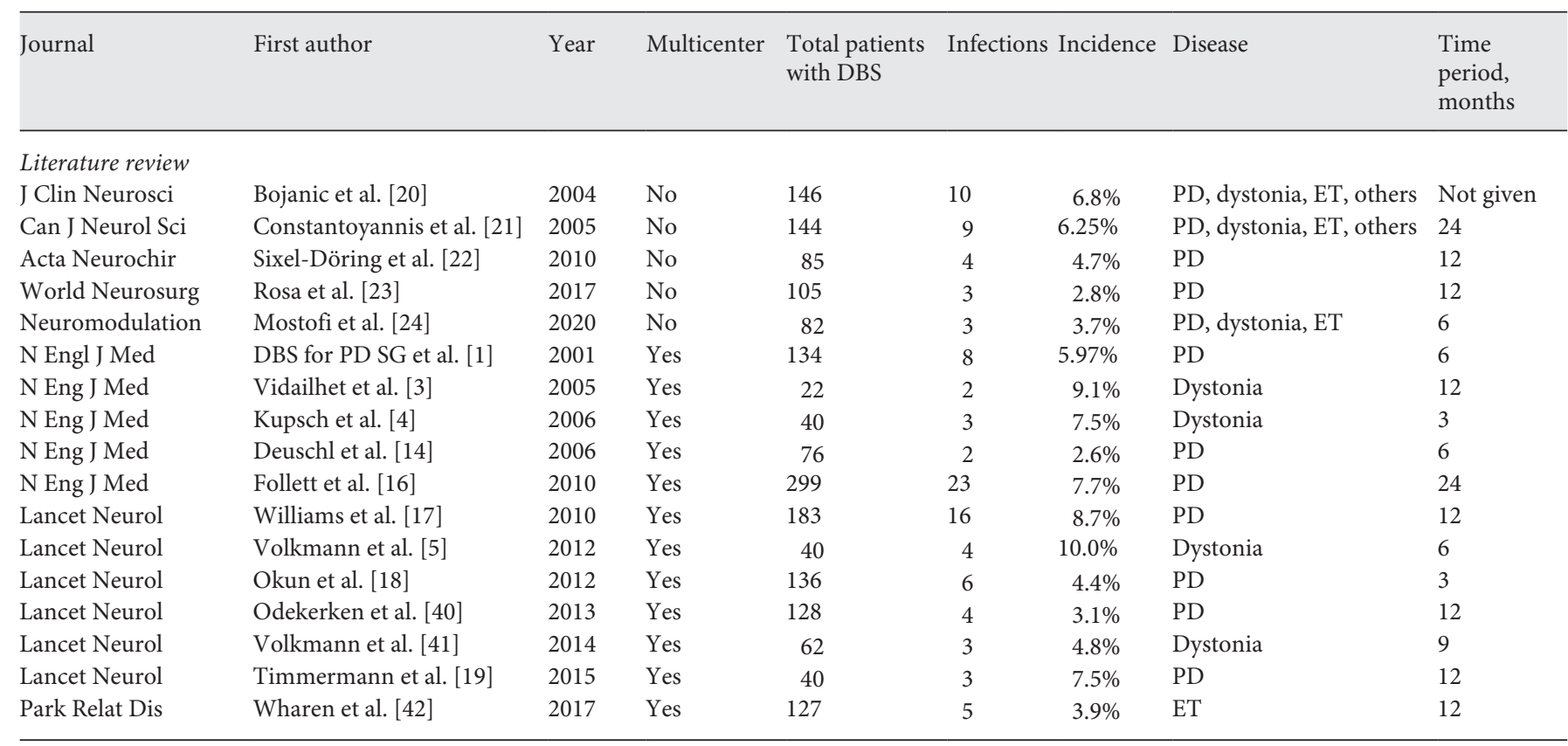

DBS, deep brain stimulation; PD, Parkinson's disease; ET, essential tremor; DBS for PD SG, deep brain stimulation for Parkinson's disease study group; all journals are conventionally abbreviated.

significant difference when comparing complications in externalized patients and patients without externalization [20, 22]. Constantoyannis et al. [21] compared very different group sizes with 118 patients and 5 infections in the non-externalized group, and only 26 patients in the externalization-group, in which 4 infections occurred. The 2 studies reporting no significant difference between externalization and non-externalized patients had more balanced groups (Sixel-Döring et al. [22]: 43 externalized patients [12 skin complications] and 42 non-externalized patients [9 skin complications]; Bojanic et al. [20]: 86 externalized patients [7 infections] and 86 non-externalized patients [3 infections]), but compared different surgical procedures. Overall, there is a little evidence concerning the infectious risk of externalization and our large single center experience supports the safety of staged surgical procedure with respect to LFP recording during externalized leads. Furthermore, we expect exclusive infections of the IPG (7/19 infection cases) to be unlikely related to externalization. Nevertheless, we have to consider as one limitation of our study that we do not have a representative control group without externalization from our center, but compare our results to the available data from the literature choosing a descriptive statistic approach due to the heterogeneous design of studies. Difference in the case number, inclusion criteria and infection definition, and reporting are possible confounders resulting in the high variability of reported infection rates. Overall, our study supports safety of LFP recordings during externalizations in a large patient cohort, especially, as the overall infection incidences are within the lower range of infection incidences in DBS reported in the literature.

\section{Important Implications for Research}

In this study, for the first time, infection incidences were assessed in relation to postoperative LFP recordings for different indications and for different DBS lead systems. Our center is a major research center for LFP recordings in human patients, with more than $70 \%$ of patients participating in recordings after informed consent. As pointed out in Table 2, there was no difference in the demographics between the patient groups participating or not in LFP recordings. Moreover, there was no difference regarding the period of externalization, whether the externalization was only used for clinical routine testing or for LFP recordings as well. This also underlines that the participation in LFP recordings does not prolong or alter treatment regimes. 
It is of high relevance for the research field that there was no significant difference in infection rates in the large patient group participating in LFP recordings. This underlines that postoperative LFP-recordings are a safe research method, as previously stated by Rosa et al. [23] in a smaller patient cohort. Our study extends this finding as we assessed infection rates not only for PD and for devices by a single manufacturer, but for all important movement disorder indications and all major manufacturers of DBS electrodes.

\section{Conclusions}

LFP recordings in DBS settings with externalized leads are not associated with an increased risk for infection as shown in our study on more than 500 patients undergoing DBS for various indications. When compared to the infection rates in the literature, our results further support the observation that externalization does not lead to a higher infection rate in DBS surgery. Overall, this study supports LFP recordings as a safe research method in human patients, when handled carefully. This is of importance since LFP research has a significant impact on future DBS development as well as understanding the pathophysiology of the underlying disorders.

\section{Acknowledgement}

We thank the Consultation Service of the Institute of Biometry and Clinical Epidemiology, Charité University Medicine Berlin for their support in the statistical analysis.

\section{Statement of Ethics}

The study was conducted ethically and in accordance with the World Medical Association Declaration of Helsinki. There was an ethics approval by the local Ethics Committee (EA1/164/20).

\section{Conflict of Interest Statement}

L.K.F., K.F., and W.J.N. report no conflict of interest. A.A.K. declares that she is on the advisory board of Boston Scientific and Mentronic and has received honoraria from Boston Scientific, Medtronic, Abbott, Teva, and Ipsen. G.H.S. received honoraria for talks for Medtronic, Abbot, and Boston Scientific.

\section{Funding Sources}

The study was funded by the German Federal Ministry of Education and Research (BMBF) grant 01GQ1802 to AAK and WJN and by the Deutsche Forschungsgemeinschaft (DFG, German Research Foundation)-Project ID 4247788381-TRR 295 grant. L.K.F. is a participant in the BIH-Charité Clinician Scientist Program funded by the Charité - Universitätsmedizin Berlin and Berlin Institute of Health.

\section{Author Contributions}

L.K.F. contributed to the conception and design of the study, to the analysis and interpretation of data, and drafted the manuscript. W.J.N. contributed to the conception and design of the study, the interpretation of data, and critically revised the manuscript for important intellectual content. K.F. contributed to the acquisition of data. G.H.S. contributed to the conception and design of the work, the data acquisition, and critically revised the work for important intellectual content. A.A.K. contributed to the conception and design of the work and revised it critically for important intellectual content. All authors approved of the final version to be published.

\section{References}

1 Deep-Brain Stimulation for Parkinson's Disease Study Group; Obeso JA, Olanow CW, Rodriguez-Oroz MC, Krack P, Kumar R, Lang AE. Deep-brain stimulation of the subthalamic nucleus or the pars interna of the globus pallidus in Parkinson's disease. N Engl J Med. 2001;345(13):956-63.

2 Putzke JD, Wharen RE Jr, Wszolek ZK, Turk MF, Strongosky AJ, Uitti RJ. Thalamic deep brain stimulation for tremor-predominant Parkinson's disease. Parkinsonism Relat Disord. 2003;10(2):81-8.

3 Vidailhet M, Vercueil L, Houeto JL, Krystkowiak P, Benabid AL, Cornu P, et al. Bilateral deep-brain stimulation of the globus pallidus in primary generalized dystonia. $\mathrm{N}$ Engl J Med. 2005;352(5):459-67.
4 Kupsch A, Benecke R, Müller J, Trottenberg T, Schneider GH, Poewe W, et al. Pallidal deep-brain stimulation in primary generalized or segmental dystonia. N Engl J Med. 2006;355(19):1978-90.

5 Volkmann J, Wolters A, Kupsch A, Müller J, Kühn AA, Schneider GH, et al. Pallidal deep brain stimulation in patients with primary generalised or segmental dystonia: 5-year follow-up of a randomised trial. Lancet Neurol. 2012;11(12):1029-38.

6 Lee DJ, Lozano CS, Dallapiazza RF, Lozano AM. Current and future directions of deep brain stimulation for neurological and psychiatric disorders. J Neurosurg. 2019;131(2):333-42.
7 Brown P. Oscillatory nature of human basal ganglia activity: relationship to the pathophysiology of Parkinson's disease. Mov Disord. 2003;18(4):357-63.

8 Kuhn AA, Williams D, Kupsch A, Limousin $\mathrm{P}$, Hariz M, Schneider G-H, et al. Eventrelated beta desynchronization in human subthalamic nucleus correlates with motor performance. Brain. 2004;127(Pt 4):73546.

9 Kuhn AA, Kupsch A, Schneider GH, Brown P. Reduction in subthalamic 8-35 Hz oscillatory activity correlates with clinical improvement in Parkinson's disease. Eur J Neurosci. 2006;23(7):1956-60. 
10 Neumann WJ, Degen K, Schneider GH, Brücke C, Huebl J, Brown P, et al. Subthalamic synchronized oscillatory activity correlates with motor impairment in patients with Parkinson's disease. Mov Disord. 2016;31(11): 1748-51.

11 Little S, Pogosyan A, Neal S, Zavala B, Zrinzo L, Hariz M, et al. Adaptive deep brain stimulation in advanced Parkinson disease. Ann Neurol. 2013;74(3):449-57.

12 Arlotti M, Marceglia S, Foffani G, Volkmann J, Lozano AM, Moro E, et al. Eight-hours adaptive deep brain stimulation in patients with Parkinson disease. Neurology. 2018; 90(11):e971-e76.

13 Schuurman PR, Bosch DA, Bossuyt PM, Bonsel GJ, van Someren EJ, de Bie RM, et al. A comparison of continuous thalamic stimulation and thalamotomy for suppression of severe tremor. N Engl J Med. 2000;342(7):4618.

14 Deuschl G, Schade-Brittinger C, Krack P, Volkmann J, Schäfer H, Bötzel K, et al. A randomized trial of deep-brain stimulation for Parkinson's disease. N Engl J Med. 2006; 355(9):896-908.

15 Vidailhet M, Vercueil L, Houeto JL, Krystkowiak P, Lagrange C, Yelnik J, et al. Bilateral, pallidal, deep-brain stimulation in primary generalised dystonia: a prospective 3 year follow-up study. Lancet Neurol. 2007;6(3):2239.

16 Follett KA, Weaver FM, Stern M, Hur K, Harris CL, Luo $\mathrm{P}$, et al. Pallidal versus subthalamic deep-brain stimulation for Parkinson's disease. N Engl J Med. 2010;362(22):2077-91.

17 Williams A, Gill S, Varma T, Jenkinson C, Quinn N, Mitchell R, et al. Deep brain stimulation plus best medical therapy versus best medical therapy alone for advanced Parkinson's disease (PD SURG trial): a randomised, open-label trial. Lancet Neurol. 2010;9(6): 581-91.

18 Okun MS, Gallo BV, Mandybur G, Jagid J, Foote KD, Revilla FJ, et al. Subthalamic deep brain stimulation with a constant-current device in Parkinson's disease: an open-label randomised controlled trial. Lancet Neurol. 2012;11(2):140-9.

19 Timmermann L, Jain R, Chen L, Maarouf M, Barbe MT, Allert N, et al. Multiple-source current steering in subthalamic nucleus deep brain stimulation for Parkinson's disease (the VANTAGE study): a non-randomised, prospective, multicentre, open-label study. Lancet Neurol. 2015;14(7):693-701.

20 Bojanic S, Sethi H, Hyam J, Yianni J, Nandi D, Joint $\mathrm{C}$, et al. Externalising deep brain electrodes: an increased risk of infection? J Clin Neurosci. 2004;11(7):732-4.

21 Constantoyannis C, Berk C, Honey CR, Mendez I, Brownstone RM. Reducing hardware- related complications of deep brain stimulation. Can J Neurol Sci. 2005;32(2):194-200.

22 Sixel-Doring F, Trenkwalder C, Kappus C, Hellwig D. Skin complications in deep brain stimulation for Parkinson's disease: frequen$c y$, time course, and risk factors. Acta Neurochir. 2010;152(2):195-200.

23 Rosa M, Scelzo E, Locatelli M, Carrabba G, Levi $\mathrm{V}$, Arlotti M, et al. Risk of infection after local field potential recording from externalized deep brain stimulation leads in Parkinson's disease. World Neurosurg. 2017;97:64-9.

24 Mostofi A, Baig F, Bourlogiannis F, Uberti M, Morgante F, Pereira EAC. Postoperative externalization of deep brain stimulation leads does not increase infection risk. Neuromodulation. 2020;24:265-71.

25 Kashanian A, Rohatgi P, Chivukula S, et al. Deep brain electrode externalization and risk of infection: a systematic review and metaanalysis. Oper Neurosurg. 2021;20(2):14150.

26 Mangram AJ, Horan TC, Pearson ML, Silver LC, Jarvis WR. Guideline for prevention of surgical site infection, 1999. Centers for Disease Control and Prevention (CDC) hospital infection control practices advisory committee. Am J Infect Control. 1999;27(2):97-6. quiz 33-4; discussion 96 .

27 Androulidakis AG, Brücke C, Kempf F, Kupsch A, Aziz T, Ashkan K, et al. Amplitude modulation of oscillatory activity in the subthalamic nucleus during movement. Eur J Neurosci. 2008;27(5):1277-84.

28 Kuhn AA, Kempf F, Brucke C, Doyle LG, Martinez-Torres I, Pogosyan A, et al. Highfrequency stimulation of the subthalamic nucleus suppresses oscillatory beta activity in patients with Parkinson's disease in parallel with improvement in motor performance. J Neurosci. 2008;28(24):6165-73.

29 Brucke C, Bock A, Huebl J, Krauss JK, Schönecker T, Schneider G-H, et al. Thalamic gamma oscillations correlate with reaction time in a Go/noGo task in patients with essential tremor. Neuroimage. 2013;75:36-45.

30 Barow E, Neumann WJ, Brücke C, Huebl J, Horn A, Brown P, et al. Deep brain stimulation suppresses pallidal low frequency activity in patients with phasic dystonic movements. Brain. 2014;137(Pt 11):3012-24.

31 Neumann WJ, Jha A, Bock A, Huebl J, Horn A, Schneider GH, et al. Cortico-pallidal oscillatory connectivity in patients with dystonia. Brain. 2015;138(Pt 7):1894-906.

32 Accolla EA, Herrojo Ruiz M, Horn A, Schneider GH, Schmitz-Hübsch T, Draganski B, et al. Brain networks modulated by subthalamic nucleus deep brain stimulation. Brain. 2016; 139(Pt 9):2503-15.

33 Huebl J, Brücke C, Merkl A, Bajbouj M, Schneider GH, Kühn AA. Processing of emo- tional stimuli is reflected by modulations of beta band activity in the subgenual anterior cingulate cortex in patients with treatment resistant depression. Soc Cogn Affect Neurosci. 2016;11(8):1290-8

34 Horn A, Neumann WJ, Degen K, Schneider GH, Kühn AA. Toward an electrophysiological "sweet spot" for deep brain stimulation in the subthalamic nucleus. Hum Brain Mapp. 2017;38(7):3377-90.

35 Neumann WJ, Horn A, Ewert S, Huebl J, Brücke C, Slentz C, et al. A localized pallidal physiomarker in cervical dystonia. Ann Neurol. 2017;82(6):912-24.

36 Lofredi R, Neumann WJ, Bock A, Horn A, Huebl J, Siegert S, et al. Dopamine-dependent scaling of subthalamic gamma bursts with movement velocity in patients with Parkinson's disease. Elife. 2018;7:e31895.

37 Neumann WJ, Schroll H, de Almeida Marcelino AL, Horn A, Ewert S, Irmen F, et al. Functional segregation of basal ganglia pathways in Parkinson's disease. Brain. 2018; 141(9):2655-69.

38 Irmen F, Horn A, Meder D, Neumann WJ, Plettig P, Schneider GH, et al. Sensorimotor subthalamic stimulation restores risk-reward trade-off in Parkinson's disease. Mov Disord. 2019;34(3):366-76.

39 Hyam JA, de Pennington N, Joint C, Green AL, Owen SL, Pereira EA, et al. Maintained deep brain stimulation for severe dystonia despite infection by using externalized electrodes and an extracorporeal pulse generator. J Neurosurg. 2010;113(3):630-3.

40 Odekerken VJ, van Laar T, Staal MJ, Mosch A, Hoffmann CF, Nijssen PC, et al. Subthalamic nucleus versus globus pallidus bilateral deep brain stimulation for advanced Parkinson's disease (NSTAPS study): a randomised controlled trial. Lancet Neurol. 2013;12(1): 37-44.

41 Volkmann J, Mueller J, Deuschl G, Kühn AA, Krauss JK, Poewe W, et al. Pallidal neurostimulation in patients with medication-refractory cervical dystonia: a randomised, shamcontrolled trial. Lancet Neurol. 2014;13(9): 875-84.

42 Wharen RE Jr, Okun MS, Guthrie BL, Uitti RJ, Larson P, Foote K, et al. Thalamic DBS with a constant-current device in essential tremor: a controlled clinical trial. Parkinsonism Relat Disord. 2017;40:18-26.

43 Kim HY. Statistical notes for clinical researchers: Risk difference, risk ratio, and odds ratio. Restor Dent Endod. 2017;42(1):72-6.

44 Green $\mathrm{H}$. Forest Plot for visualisation of multiple odds ratios MATLAB Central File Exchange. 2020. Available from: https://www. $\mathrm{mathworks.com/matlabcentral/}$ fileexchange/71020-forest-plot-for-visualisation-of-multiple-odds-ratios. 